Apidologie, 1988, 19 (3), 275-290

\title{
ANALYSE POLLINIQUE DES MIELS DU NORD-OUEST DE LA PROVINCE DE BUENOS AIRES (RÉPUBLIQUE ARGENTINE)
}

\author{
María C. TELLERIA \\ Museo de Ciencias Naturales - 1900 La Plata (République Argentine)
}

\begin{abstract}
RÉSUMÉ
Cinquante-trois échantillons de miel, recueillis dans le Nord-Ouest de la Province de Buenos Aires de 1981 à 1983, ont été analysés du point de vue pollinique. On a déterminé 90 types morphologiques, la plupart d'entre eux au niveau spécifique. La caractéristique de ces miels est leur richesse en pollen de Légumineuses et de Composées ainsi qu'en pollen d'Eucalyptus sp., Crucifères, Echium plantagineum, Mentha sp., Conium sp. et Ammi sp. On remarque la prédominance du pollen des espèces herbacées et la faible représentation du pollen des espèces arborescentes autochtones, de même que des indicateurs de miellat. Les différences dans la teneur en pollen des échantillons provenant de divers districts pourraient être dues aux différences de conditions édaphiques. La plupart des échantillons renferment entre 20000 et 100000 grains par $10 \mathrm{~g}$ de miel.
\end{abstract}

\section{INTRODUCTION}

L'analyse pollinique des miels donne une information précise sur les principales plantes mellifères et permet de caractériser les miels par leur origine botanique ou géographique. Elle apporte des informations importantes sur le comportement de butinage des abeilles. Par ailleurs, la teneur en pollen des miels permet de contrôler leur qualité, augmentant ainsi leur valeur économique.

Malgré l'importance du miel comme aliment et sa valeur commerciale comme produit exportable, en Argentine le spectre pollinique des miels n'est pas bien connu (CosTA DE BRINGAS, 1982). La plupart des études réalisées jusqu'à présent n'ont considéré que les aspects chimiques (Gamero et al., $1969 ; 1971 ; 1972)$. On ne possède également pas beaucoup d'informations sur la valeur apicole des plantes. Les renseignements dont on dispose correspondent en général à des observations effectuées par les apiculteurs sur le terrain qui n'ont pas été confirmées par l'analyse pollinique.

La méconnaissance du sujet et son importance économique justifient le présent travail qui a pour objet d'effectuer l'analyse pollinique qualitative et 


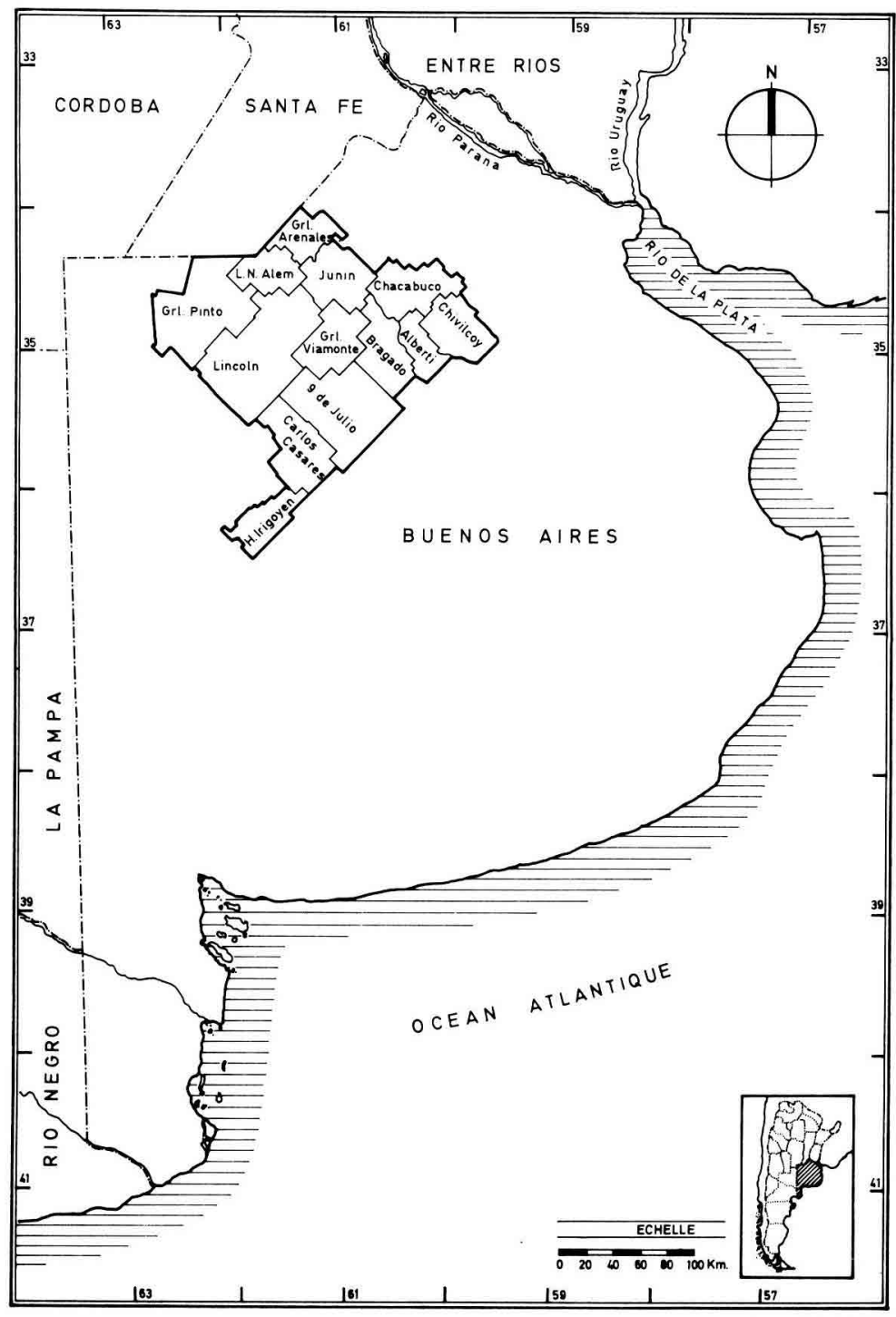

FIG. 1. - Localisation de l'aire d'étude.

FIG. 1. - Location of the area studied. 
quantitative des miels du Nord-Ouest de la Province de Buenos Aires. L'aire sélectionnée (Fig. 1) est une des zones les plus productives de miel dans la province citée ; elle comprend au total 13 districts.

Elle est située dans le domaine phytogéographique de la pampa (CABRERA, 1963) et sa végétation caractéristique est la steppe de graminées, qui se trouve d'ailleurs presque totalement détruite par l'action de l'homme. Les cultures les plus importantes du point de vue apicole sont : Helianthus annuus L., Medicago sativa L., et Zea mays L. Parmi les autres plantes cultivées d'intérêt apicole, on peut citer celles qui sont utilisées comme arbres d'alignement : Acacia Mill., Eucalyptus L'Herit., Populus alba L., Casuarina cuninghamiana Miq., et Melia azedarach $\mathbf{L}$.

\section{MATÉRIEL ET MÉTHODES}

\section{Matériel de référence}

Les préparations de référence ont été réalisées pour déterminer avec précision les grains de pollen contenus dans les miels. Dans ce but on a recueilli des plantes en fleurs dans l'aire étudiée. Ce matériel a été déterminé et incorporé à l'herbier de la Division des Plantes vasculaires du Musée de La Plata. On a fait deux préparations par espèce, l'une selon la technique de WoDEHouse (1935), et l'autre à partir de matériel acétolysé (Erdrman, 1969). Dans les deux cas on a utilisé la glycérine gélatinée comme milieu de montage. Les grains de Composées et de Malvacées ont été chlorinés et montés dans l'hydrate de chloral.

\section{Récolte et préparations des échantillons de miel}

La récolte a été effectuée pendant les mois de décembre à février des années 1981 à 1983. Cinquante-trois échantillons ont été prélevés: Alberti 6, L.N. Alem 2, Gral. Arenales 2, Bragado 2, Carlos Casares 5, Chacabuco 4, Chivılcoy 7, H. Irigoyen 5, 9 de Julio 4, Junín 6, Lincoln 4, Gral. Pinto 3, Gral. Viamonte 3 (Fig. 1).

Pour effectuer l'analyse qualitative et quantitative, on a suivi les techniques proposées par LouvEAuX et al. (1978). Cependant, avant de réaliser l'analyse qualitative on a identifié les types polliniques en utilisant le microscope photonique et le microscope électronique à balayage. Dans ce dernier cas les grains obtenus par dilution et centrifugation des miels, sont lavés au moins deux fois dans l'alcool absolu, puis montés sur plaques de cuivre sous la loupe binoculaire.

Pour chaque miel 200 grains de pollen, ainsi que les indicateurs de miellat, ont été comptés. Les résultats du dénombrement ont été classés en groupes d'après leur fréquence (op. cit.) : pollen dominant (plus de $45 \%$ du pollen total), pollen secondaire (16-45\%), pollen de moindre importance (3-15\%) et pollen mineur $(<3 \%)$.

La fréquence d'apparition de chaque type morphologique correspond dans ce cas au nombre de fois qu'il apparait dans l'ensemble des échantillons.

On a calculé les indices de miellat (HDE, honey dew elements); ils sont indiqués par le quotient HDE/P ( $P$ : pollen).

La richesse en grains de pollen pour $10 \mathrm{~g}$ de miel a été déterminée à partir du dénombrement dans un hématimètre. Les résultats sont exprimés comme suit : groupe I (20000 ou moins), groupe II (20 000 $100000)$, groupe III (100 000-500 000), groupe IV (500 000-1 000 000) et groupe V (plus de 1000000 de grains). 


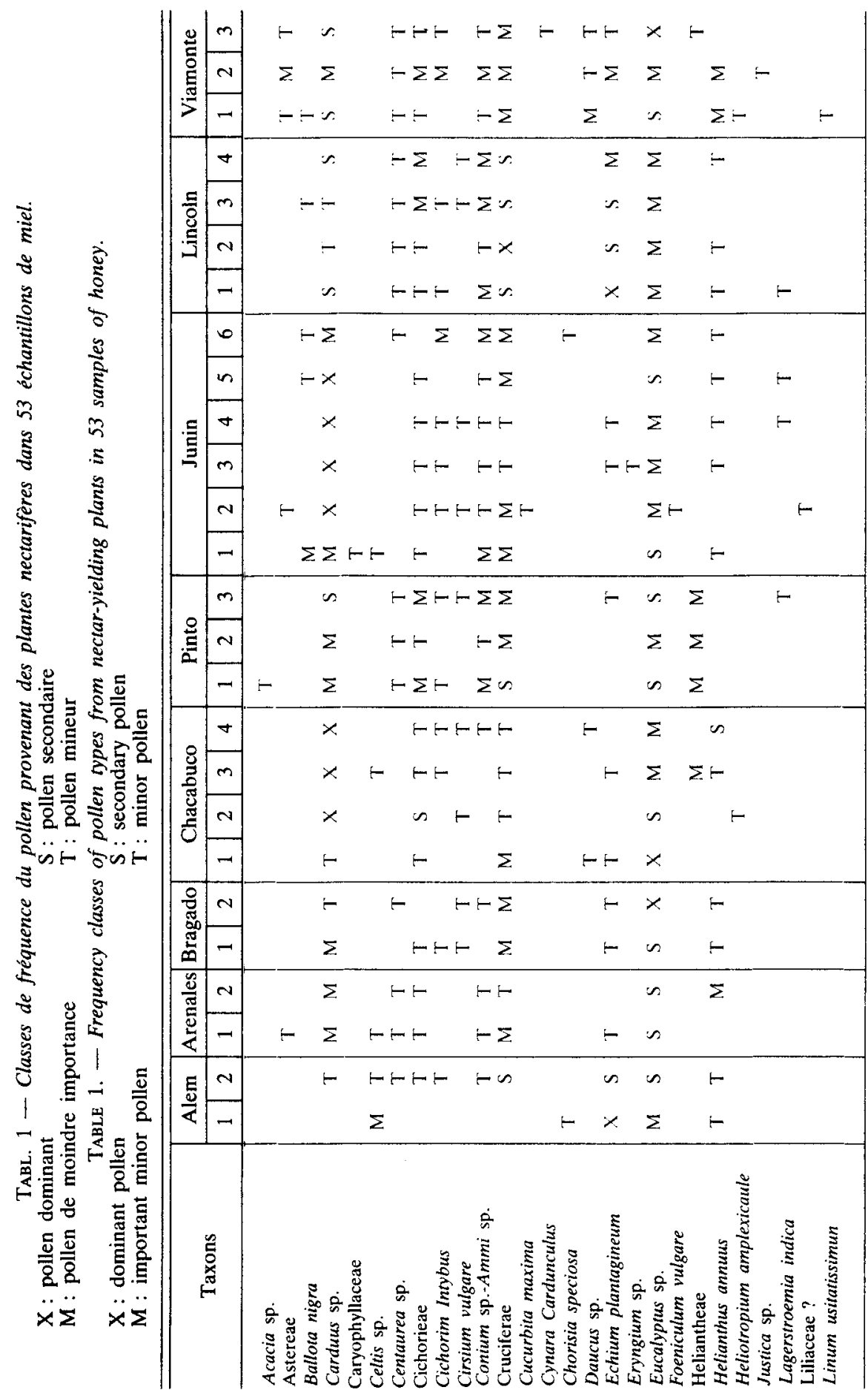




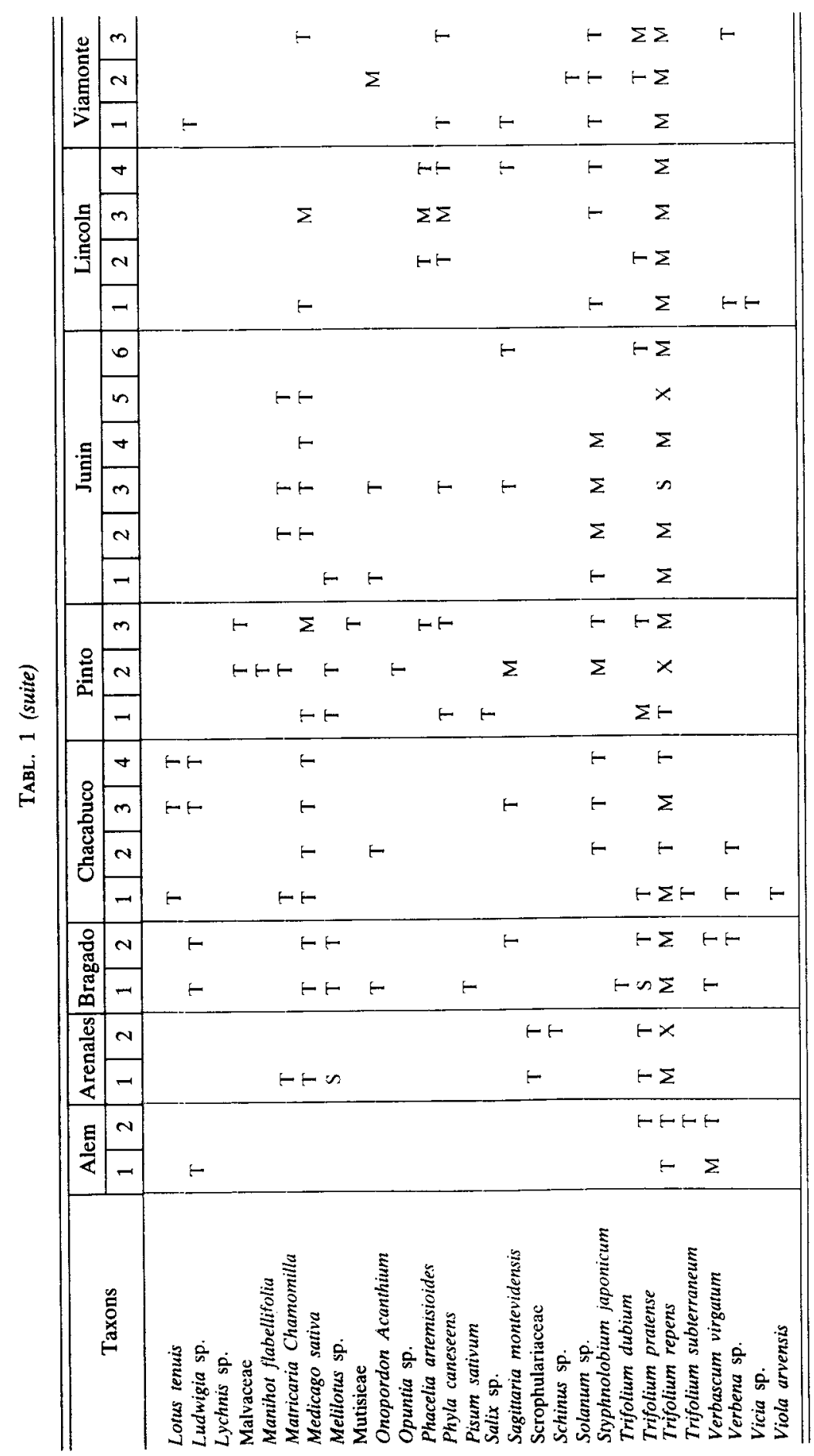




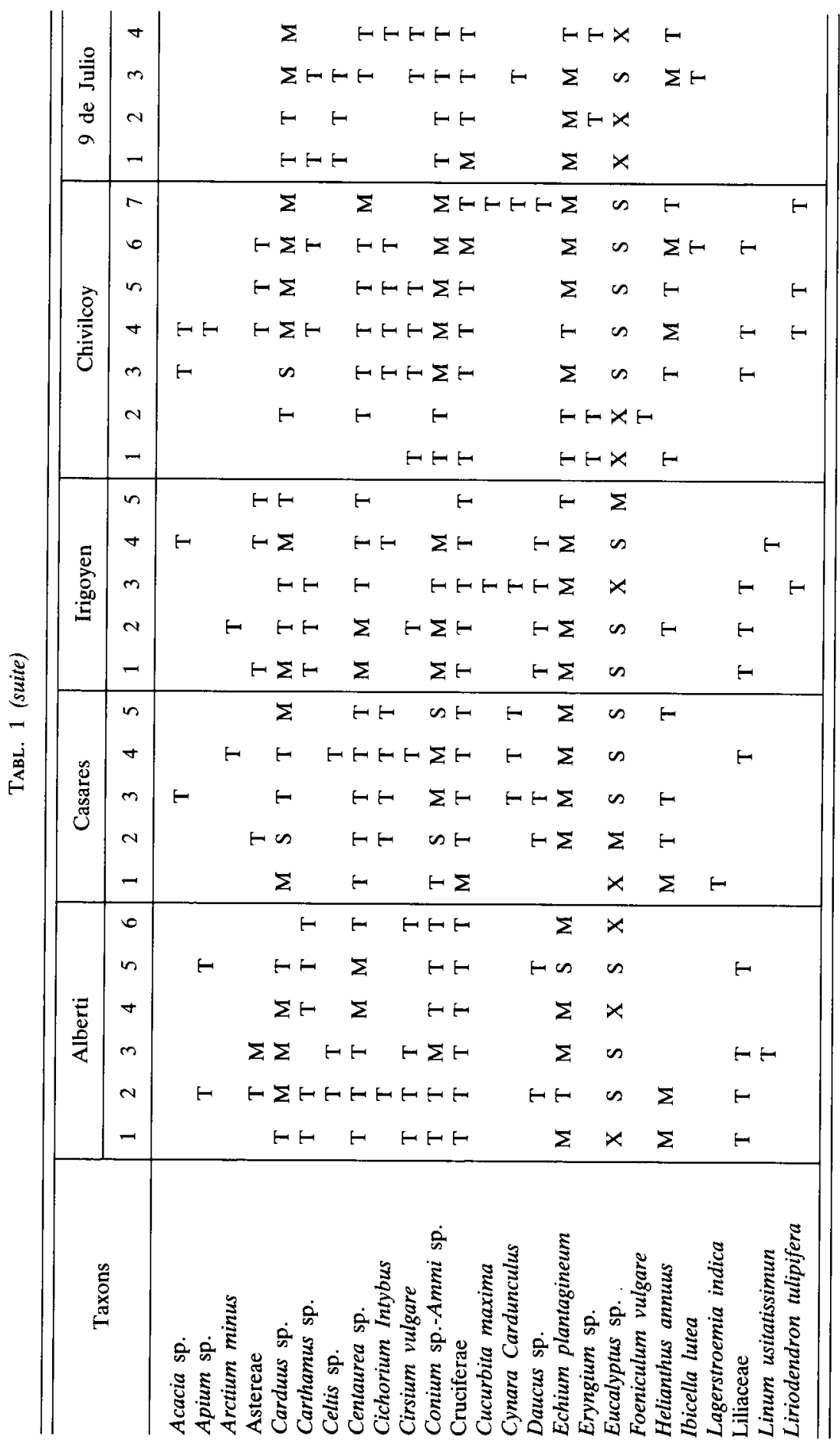




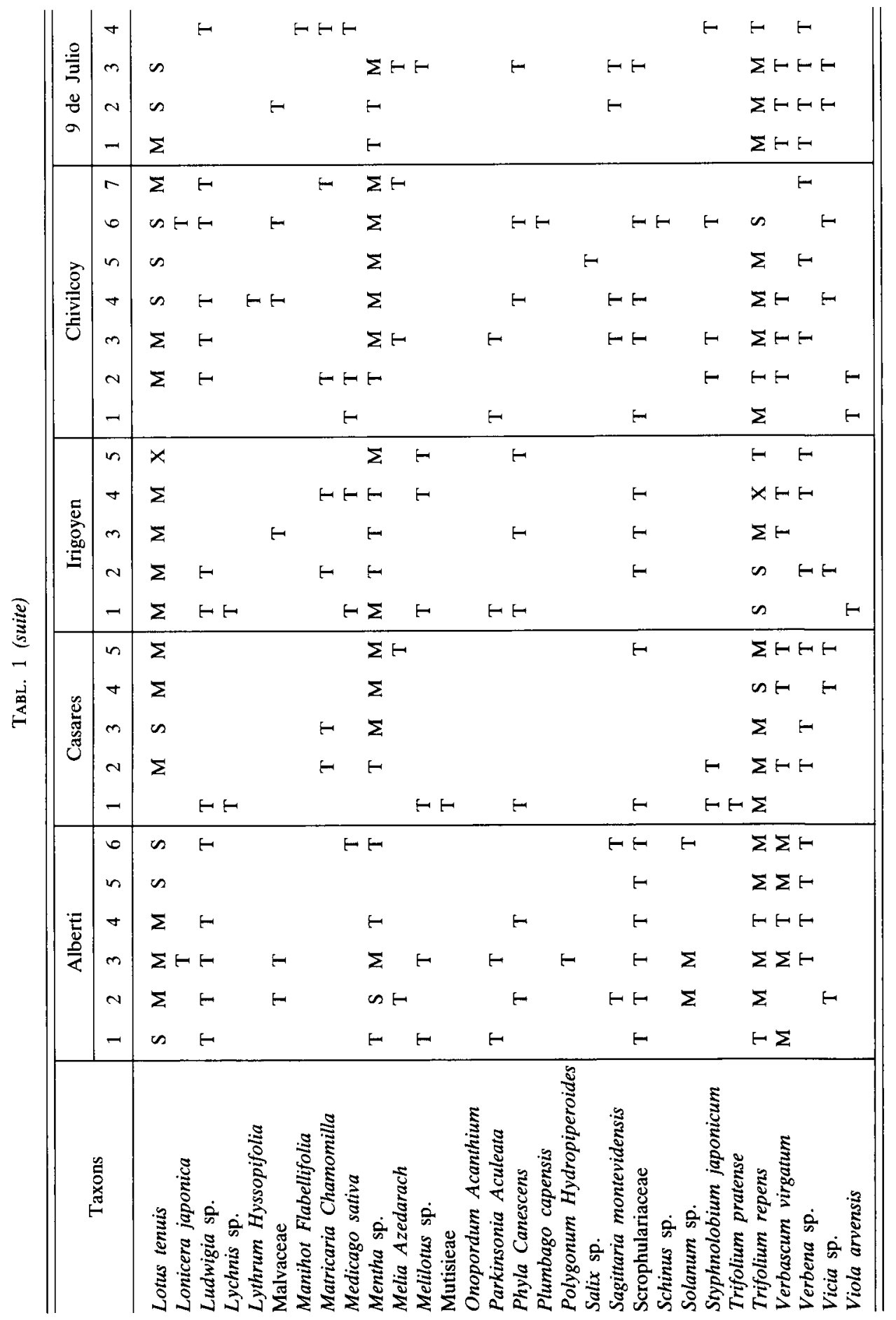




\section{RÉSULTATS}

On a identifié 90 types morphologiques (Fig. 3): 44 au niveau de l'espèce, 30 de la tribu, 11 de la famille et 1 de l'ordre (Pteridophyta).

La figure 2 montre le rapport entre le nombre de types morphologiques et le nombre d'échantillons dans lesquels ils apparaissent.

Le tableau 1 représente les classes de fréquence de chaque plante nectarifère dans les différents échantillons. Il donne une vision générale des espèces qui contribuent le plus à l'élaboration des miels dans l'aire étudiée. Il permet aussi de reconnaître les miels monofloraux; ils proviennent de : Eucalyptus (12 échantillons), Carduus L. (7 échantillons), Trifolium repens L. (4 échantillons), Cruciferae (2 échantillons) et Lotus tenuis Waldst. et Kit. (1 échantillon).

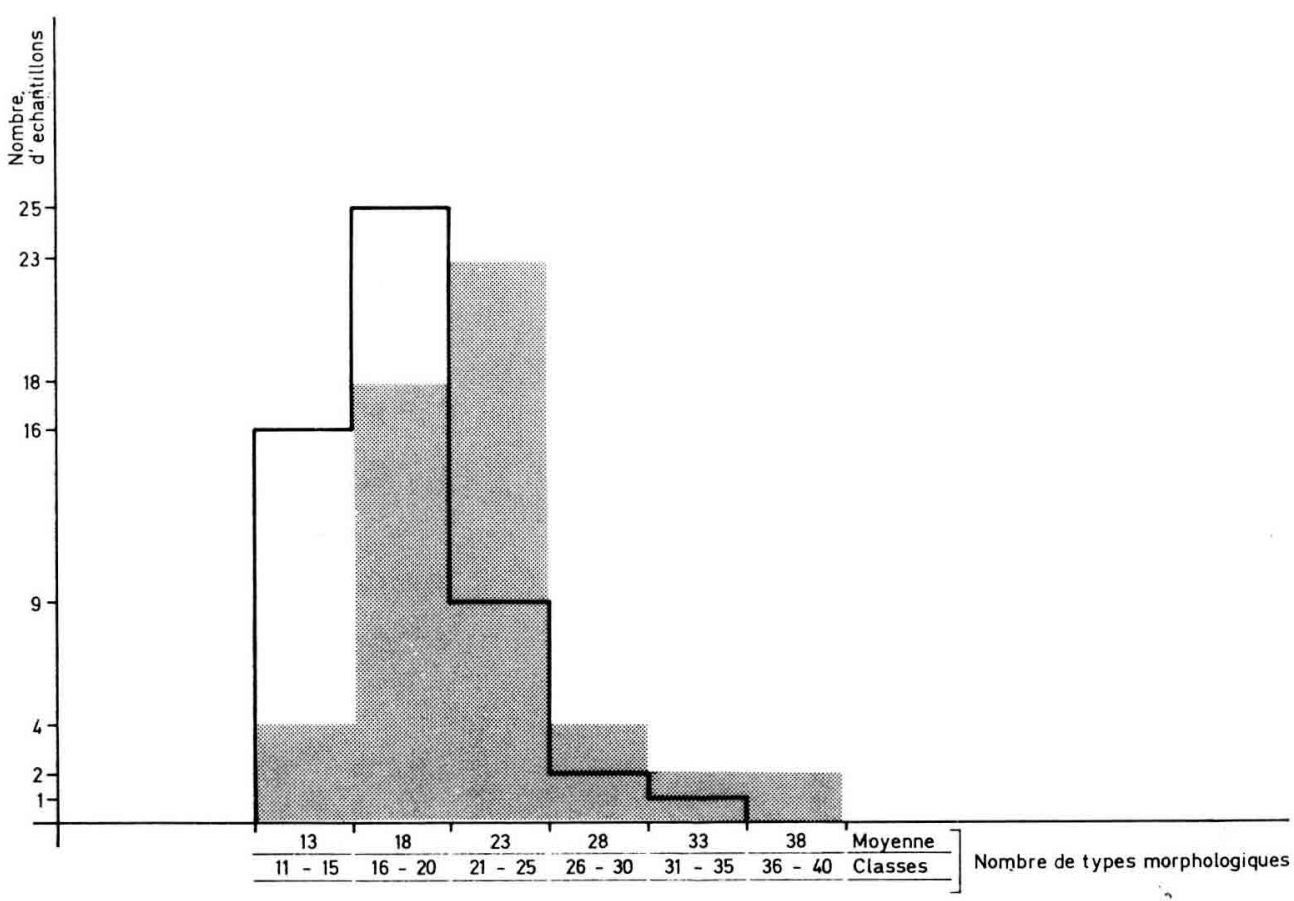

Fig. 2. - Nombre des différentes formes de pollen dans les miels.

(La zone ombrée correspond aux types morphologiques provenant des plantes nectariferes).

Fig. 2. - Number of different pollen types in honey samples.

(The dark area represents the morphological types from nectar-yielding plants). 


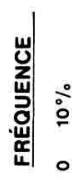

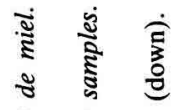

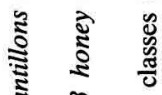

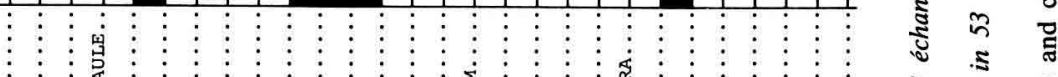

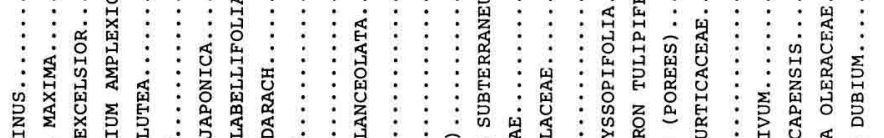

กิ ฏ

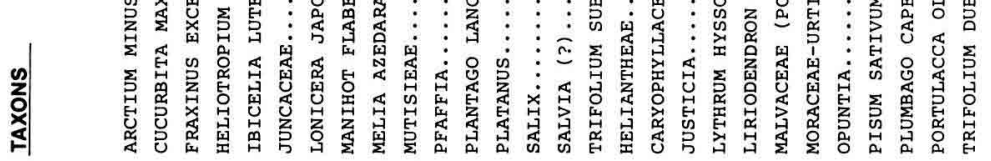

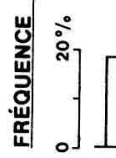

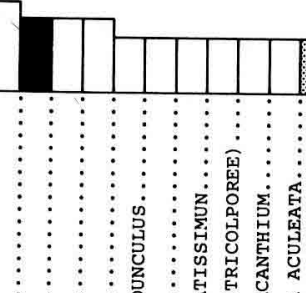

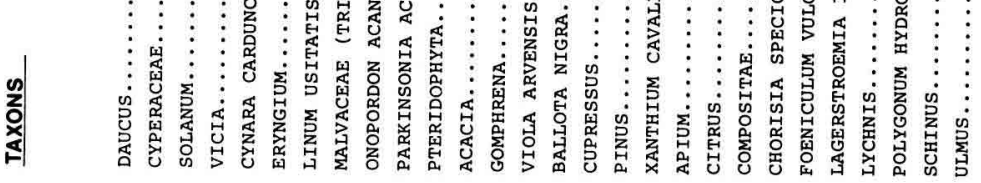
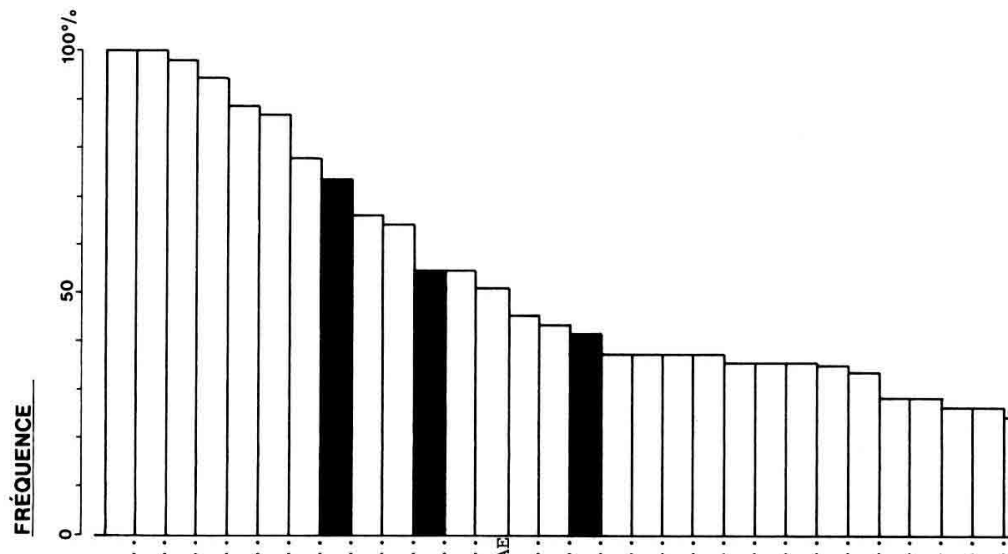

¿

हैँ

\& \&

¿

尊

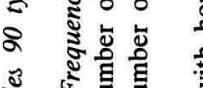

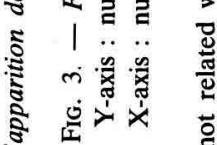

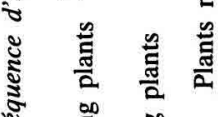

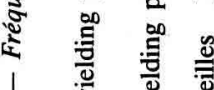

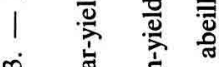

ن

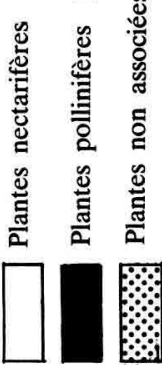


La figure 3 représente la fréquence d'apparition des 90 taxa déterminés. On peut observer que Trifolium repens et Eucalyptus sont présents dans la totalité des échantillons. Il existe 13 taxa qui dépassent $50 \%$; la plupart sont nectarifères, 77 types pollinique se manifestent à une fréquence inférieure à $50 \%$.

La figure 4 synthétise les résultats de l'analyse quantitative.

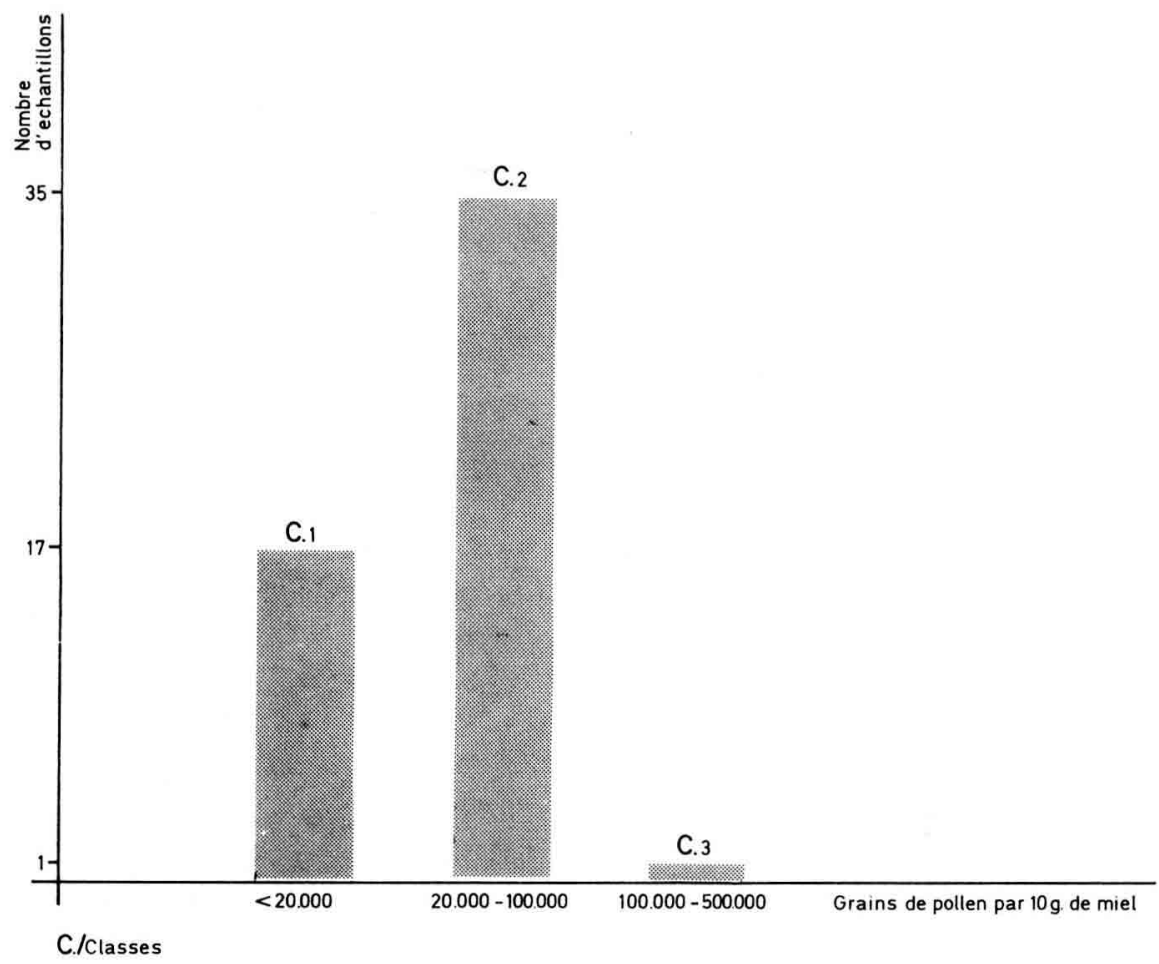

Fic. 4. - Nombre des grains de pollen contenus dans $10 \mathrm{~g}$ de miel.

Fig. 4. - Number of pollen grains within $10 \mathrm{~g}$ of honey.

Y-axis : number of samples.

$\mathrm{X}$-axis : pollen grains per $10 \mathrm{~g}$ of honey. 


\section{DISCUSSION}

\section{Origine botanique}

La détermination et le dénombrement des différents types morphologiques présents dans les miels ont permis la caractérisation botanique de ceux-ci. La haute représentativité des Composées et des Légumineuses, ainsi que l'abondance du pollen d'Eucalyptus sp., sont les caractères remarquables de ces miels.

Parmi les Composées, le groupe des "chardons » occupe une place très importante : Carduus sp., Carthamus sp., Cirsium vulgare (Savi.) Ten., Cynara Cardunculus L. et Onopordon Acanthium L. On peut ajouter : Ambrosia L., Cichorium Intybus L., Helianthus annuus, Matricaria Chamomilla L., Xanthium cavallinesii Schouw., et des genres non déterminés appartenant aux Astereae, Chichorieae, Heliantheae et Mutisieae.

Dans les Légumineuses, les " trèfles » ont un rôle prépondérant. On a déterminé : Trifolium dubium Sibthorp, T. pratense L., T. repens, T. subterraneum L., Lotus tenuis, Melilotus Mill., et Medicago sativa, ainsi que : Acacia sp., Parkinsonia aculeata L., Pisum sativum L., Styphnolobium japonicum (L.) Schott. et Vicia L.

Les Crucifères constituent aussi un élément caractéristique de ces miels. Malgré leur homogénéité, du point de vue palynologique, on a reconnu : Brassica L., Raphanus sativus L. et Rapistrum rugosum (L.) All.

Les grains de pollen qui se trouvent en quantités inférieures à $15 \%$ (pollen de moindre importance et pollen mineur) ne sont pas considérés, en général, dans la caractérisation des miels. Cependant ils nous permettent de mieux connaître les préférences des abeilles et nous apportent occasionnellement des renseignements très importants sur le milieu d'où proviennent les miels (proximité de cours d'eau, cultures économiquement importantes, etc.). Dans cette catégorie on trouve des pollens de plantes nectarifères ou pollinifères ou de plantes non associées aux abeilles.

Parmi les plantes nectarifères représentées dans les miels (Tabl. 1) figurent: Acacia sp., Apium L., Arctium minus, Astereae, Ballota nigra L., Carthamus sp., Caryophyllaceae, Celtis Taurn., Cichorieae, Cichorium Intybus, Citrus L., Cruciferae, Cucurbita maxima Duch., Chorisia speciosa St. Hill., Daucus L., Echium plantagineum L., Eryngium L., Foeniculum vulgare Hill., Heliantheae, Heliotropium amplexicaule Vahl, Ibicella lutea (Lindl.) Van Eselt, Justicia L., Liliaceae ?, Linum usitatissimum L., Liriodendron tulipifera L., Ligustrum L., Lonicera japonica Thunb., Lychnis (Tourn.) L., Ludwigia L., Lythrum hyssopifolia L., Malvaceae, Manihot flabellifolia Pohl., Matricaria 
Chamomilla, Medicago sativa, Melia Azedarach, Mutisieae, Onopordon Acanthium, Opuntia Mill., Phacelia artemisioides Griseb., Phyla canescens (H.B.K.) Greene, Polygonum hydropiperoides Mich., Plumbago capensis L., Portulacca oleracea L., Salix (Tourn.) L., Sagittaria montevidensis Cham. et Schlech., Scrophulariaceae, Solanum L., Tamarix gallica L., Trifolium dubium, T. subterraneum, Ulmus (Taurn.) L., Verbascum virgatum Stokes, Verbena L., Vicia sp. et Viola arvensis Murray.

Parmi les plantes pollinifères, anémophiles en général, on a trouvé : Ambrosia sp., Casuarina cuninghamiana, Cyperaceae, Chenopodiaceae-Amaranthaceae, Fraxinus excelsior, Gomphrena L., Gramineae, Juncaceae, Moraceae-Urticaceae, Pfaffia Mart., Plantago lanceolata L., Platanus (Tourn.) L., Xanthium cavallinesii et Zea mays.

Parmi les plantes sans rapport avec les abeilles, on a déterminé des Conifères (Pinus (Tourn.) L. et Cupressus Tourn. ex L.) et des fougères. Les abeilles pourraient utiliser pour leur nourriture tant le pollen que les spores de ces plantes pendant les périodes de pénurie de fleurs (Percival, 1947), mais en raison de la sous-représentation de ces groupes dans les échantillons analysés on pense à une contamination.

En ce qui concerne les indices de miellat le rapport $\mathrm{HDE} / \mathrm{P}$ a toujours été inférieur à 1,5. Ces éléments sont très fréquents dans les miels provenant de zones comportant de grandes étendues boisées, caractéristique qui n'appartient pas à notre aire d'étude.

On a trouvé que la plupart des échantillons renfermaient entre 20000 et 100000 grains de pollen par 10 grammes de miel (Fig. 4).

\section{Origine géographique}

Du point de vue géographique, ces miels pourraient être caractérisés selon la combinaison des types morphologiques dominants et secondaire, comme le propose Maurizio (1971). Dans ce cas, ce serait une combinaison du pollen de Carduus sp., Centaurea sp., Conium sp.-Ammi sp., Cruciferae, Echium plantagineum, Eucalyptus sp., Helianthus annuus, Lotus tenuis, Melilotus sp., Trifolium pratense et $T$. repens.

Cependant on a trouvé des différences remarquables dans le spectre pollinique des échantillons provenant de divers districts. On remarque des différences fondamentales au niveau de quelques types morphologiques dominants et secondaires. Elles correspondent à différentes conditions édaphiques qui se reflètent dans la végétation. Ainsi si l'on compare les districts de Chivilcoy, Alberti, 9 de julio, Carlos Casares et Hipólito Irigoyen, avec les 
autres, la caractéristique la plus remarquable est la présence de Lotus tenuis et Mentha sp., l'absence de Trifolium pratense et la moindre abondance des Crucifères.

Quant à Centaurea sp., Conium sp.-Ammi sp., Eucalyptus sp., Helianthus annuus et Trifolium repens leur présence et leur abondance n'ont pas présenté de variations remarquables dans les échantillons analysés (Tabl. 1).

On peut constater que les miels du Nord-Ouest de la Province de Buenos Aires sont caractérisés non seulement par un ensemble de types morphologiques, mais présentent encore trois aspects fondamentaux :

- prédominance du pollen des espèces herbacées, principalement introduites ou envahissantes ;

- absence du pollen des espèces arborescentes autochtones (ou seulement présence à l'état de traces) ;

— indicateurs de miellat très peu ou non représentés.

Il est intéressant de comparer le spectre pollinique obtenu avec d'autres qui existent dans plusieurs régions du monde. Il en résulte que les miels étudiés partagent quelques types morphologiques avec ceux de l'Europe occidentale et de l'Amérique du Nord, spécialement des Papilionacées. Néanmoins, ces miels ne peuvent pas être confondus. Le pollen de Rosacées, des Labiées (nombreuses espèces), des Ericacées, des espèces arborescentes, de même que les indicateurs de miellat, si communs dans les miels européens (Louveaux, 1968), sont absents des miels analysés.

Il en est de même pour les Rosacées, Rhamnacées, Composées du type Aster et pour de nombreuses espèces arborescentes présentes dans les miels américains (LouveAuX, 1968 ; LIEUX, 1972).

Reçu pour publication en octobre 1986. Accepté pour publication en mars 1988.

\section{REMERCIEMENTS}

Je veux exprimer ma reconnaissance au $D^{r}$ Marta A. MoRbelli pour avoir dirigé ce travail, à la Commission de Recherches Scientifiques de la Province de Buenos Aires pour l'aide financière qui a permis sa réalisation, au Musée de Sciences Naturelles de La Plata pour m'avoir fourni un laboratoire d'accueil, aux Coopératives Apicoles et aux apiculteurs pour la fourniture des échantillons.

J'exprime ma reconnaissance aux $D^{\text {rs }}$ Elias R. DE la Sota, Oscar Zubietta, Lic. Maria T. CAstro et aux Ing. Agr. Luis G. Cornejo et Rafael Boggio RonCERos. 


\section{SUMMARY \\ PALYNOLOGICAL ANALYSIS OF HONEYS FROM THE NORTH-WEST OF THE PROVINCE OF BUENOS AIRES}

53 samples of honey from the North-West region of the province of Buenos Aires (Fig. 1) were analyzed using the techniques proposed by Louveaux et al. (1978). The morphological types were determined using a collection of reference pollens.

90 morphological types corresponding to different taxonomic levels, most of them to a species level, were identified. From the total 73 belonged to nectar plants, 14 to pollen plants and 3 to plants that are not connected with bees. Their frequency of occurrence corresponds to the number of times each of them occur in the samples (Fig. 3). Figure 2 shows the ratio between the number of morphological types and the number of samples in which they occur. Honeydew elements (HDE) were calculated ; they are indicated by the ratio $\mathrm{HDE} / \mathrm{P}(\mathrm{P}=$ pollen). This ratio was always below 1.5 .

27 samples had a mixed floral origin, the other were monofloral from Eucalyptus (12 samples), Carduus (7 samples), Trifolium repens (4 samples), Cruciferae (2 samples) and Lotus tenuis (1 sample). Table 1 gives the frequency classes of nectar plants in the different samples.

The quantitative analysis was carried out in a haemocytometer. It showed that most of samples contained from 20.000 to 100.000 grains per $10 \mathrm{~g}$ of honey (Fig. 4).

The analyses also permitted botanic characterization of the honey. It was composed largely of Compositae and Leguminosae but also contained a great abundance of Eucalyptus and Cruciferae pollen (Brassica L., Raphanus sativus and Rapistrum rugosum).

From the geographic point of view these honeys could be characterized according to the combination of dominant and secondary morphological types (MAURizio, 1971). In this case it is a pollen combination of Carduus sp., Centaurea sp., Conium sp., Ammi sp., Cruciderae, Echium plantagineum, Eucalyptus sp., Helianthus annuus, Lotus tenuis, Melilotus sp., Mentha sp., Trifolium pratense and T. repens. However, coming from different districts of the Province, differences were found in some of the dominant and secondary types in samples; they are probably due to edaphic conditions (Tabl. 1).

\section{ZUSAMMENFASSUNG}

\section{POLLENANALYSE VON HONIGEN AUS DEM NORDWESTEN DER PROVINZ BUENOS AIRES (REPUBLIK ARGENTINIEN)}

Es wurden 53 Honigproben aus dem Nordwesten der Provinz Buenos Aires nach der von Louveaux et al. (1978) angegebenen Methode analysiert (Fig. 1). Die Pollenkörner wurden nach einer Sammlung von Referenzpollen bestimmt.

Es konnten 90 morphologische Typen bestimmt werden, meist bis zu dem Niveau der Spezies (Fig. 3). Von diesen Pollenformen gehörten 73 zu nektarspendenden Pflanzen, 14 zu Pollenpflanzen und drei zu solchen, die keine Beziehungen zu Bienen haben. Ihre Häufigkeit (Frequenz) wird danach angegeben, wie oft jeder Typ in den Proben gefunden wurde (Fig. 3). Fig. 2 zeigt die Beziehungen zwischen der Anzahl morphologischer Typen und der Zahl der Proben, in denen sie auftraten. Außerdem wurden die Honigtauelemente (HDE) bestimmt. Der Honigtauanteil wird in Form eines Quotienten HDE/P $(\mathrm{P}=$ Pollen) angegeben. Diese Quotient war immer kleiner als 1.5 .

27 Proben stammten von mehreren Blütensorten, der Rest war monofloral von Eucalyptus (12 Proben), Carduus (7 Proben), Trifolium repens (4 Proben), Cruciferae (2 Proben) und Lotus tenuis (1 Probe). Tab. 1 zeigt die Häufigkeitsklassen der Honigpflanzen in den verschiedenen Proben. 
Die quantitative Pollenanalyse, ausgeführt durch Auszählung in einem Hämozytometer, ergab bei den meisten Proben einen Pollengehalt zwischen 20.000 und 100.000 Körnern pro $10 \mathrm{~g}$ Honig (Fig. 4).

Die analysierten Honige sind durch ein häufiges Auftreten von Compositen und Leguminosen, aber auch von Eucalyptus und verschiedenen Cruciferen (Brassica sp., Raphanus sativus, Rapistrum rugosum) charakterisiert.

In geographischer Hinsicht können die Honige durch eine bestimmte Kombination von Leit- und Begleitpollen charakterisiert werden (MAURIzIo, 1971). Im vorliegenden Fall ist es eine Kombination von folgenden Pollen : Carduus sp., Centaurea sp., Conium sp., Ammi sp., Cruciferae, Echium plantagineum, Eucalyptus sp., Helianthus annuus, Lotus tenuis, Melilotus sp., Mentha sp., Trifolium pratense und $T$. repens. Es wurden aber Unterschiede bei den Leit- und Begleitformen in den verschiedenen Distrikten der Provinz gefunden (Tabl. 1), vermutlich infolge unterschiedlicher edaphischer Bedingungen.

\section{RÉFÉRENCES BIBLIOGRAPHIQUES}

Cabrera A.L., 1963. - Flora de la Provincia de Buenos Aires. Parte 1. Colección Cientifica del INTA.

Costa de Bringas M.C., 1982. - Contribución al conocimiento de la flora melífera de la Provincia de Córdoba. I. Departamento Río Segundo. Bol. Soc. Argent. Bot., 21 (1-4), 247-258.

Erdtman G., 1969. - Handbook of Palynology. Muskgaard, Copenhagen, 486 p.

Gamero A., Cornejo L. y Tomasevich L., 1969. - Caracterización de las mieles de la Provincia de Buenos Aires. Producción Animal, 2 (1), 1-34.

Gamero A., Cornejo L. y Schminke E., 1971. - Caracterización de las mieles de la Provincia de Buenos Aires. Zona II. Producción Animal, 2 (4), 133-158.

Gamero A., Cornejo L. y Schminke E., 1972. - Caracterización de las mieles de la zona sur de la Provincia de Buenos Aires. Producción Animal, 3 (1), 1-26.

Lieux M., 1972. - A melissopalynological study of 54 Louisiana (U.S.A.) honeys. Rev. Palaeobot. Palynol., 13, 95-124.

Louveaux J., 1968. - L'Analyse pollinique des miels. Traité de Biologie de l'abeille. Ed. R. Chauvin, t. 3, Masson, Paris, 325-362.

Louveaux J., Maurizio A. and Vorwhol G., 1978. - Methods of Melissopalynology by International Commission for Bee Botany of IUBS. Bee World, 59, 139-157.

Maurizio A., 1971. - Le spectre pollinique des miels luxembourgeois. Apidologie, 2 (3), 221-238.

Percival M., 1947. - Pollen collection by Apis mellifera. New Phytol., 46, 142-173.

Wodehouse R.P., 1935. - Pollen Grains. Mc. Graw-Hill Book Co., New York, London, 574 p. 